\title{
Effects of Power Amplifier Nonlinearities on the Radar Ambiguity Function
}

\author{
Dylan Eustice ${ }^{1}$, Charles Baylis ${ }^{1}$, Lawrence Cohen ${ }^{2}$, and Robert J. Marks II ${ }^{1}$ \\ ${ }^{1}$ Wireless and Microwave Circuits and Systems Program \\ Department of Electrical and Computer Engineering \\ Baylor University \\ Waco, TX, USA \\ ${ }^{2}$ Radar Division \\ United States Naval Research Laboratory \\ Washington, DC, USA
}

\begin{abstract}
The radar ambiguity function describes the possibility of errors in range and Doppler detection for targets of certain range-Doppler combinations. Nonlinear power amplifiers in radar transmitters provide many undesirable effects, including spreading of the radar spectrum. Based on the known impacts of the power amplifier nonlinearities on the waveform and spectrum that is transmitted, this paper assesses the related impacts on the ambiguity function and, therefore, on the estimation precision capabilities of the radar. Simulation results for nonlinear microwave power-amplifier transistor models are presented to illustrate the theory that is presented. Simulations using a Gaussian Barker code implementation show that amplifier nonlinearities cause widening of ambiguity content in the Doppler dimension, widening of range sidelobes, and deepening of range and Doppler nulls. The theory and observations presented in this paper are expected to find significant application in radar waveform optimization to provide desired ambiguity-function properties while maintaining spectral compliance.
\end{abstract}

Keywords - ambiguity function, power amplifiers, spectrum engineering, estimation

\section{INTRODUCTION}

The ambiguity function describes the ability of a radar waveform $x(t)$ to precisely determine the range and Doppler of a target. It is defined as follows [1]:

$$
\chi(\tau, u)=\int_{t=-\infty}^{\infty} x(t) x^{*}(t-\tau) e^{-j 2 \pi u t} d t
$$

where $\tau$ and $u$ are the range and Doppler errors, respectively, relative to the actual range and Doppler of a measured target. In radar transmitters, the waveform is distorted by the nonlinear power amplifier before transmission, causing a resultant change in the ambiguity function of the waveform. This change is of interest to radar designers and operators, because it is in essence a change in the radar system's range and Doppler estimation capabilities due to power amplifier nonlinearities. Optimization for spectral performance will impact the ambiguity function, and vice versa. Power amplifier nonlinearities that cause spectral spreading are examined for their related impact on the ambiguity function.

Significant attention is provided in the literature to the spectral spreading result of amplifier nonlinearities. De Graaf examines the impact of a power amplifier on the spectra of radar waveforms, and includes comparisons of the amplifier output waveform spectrum to spectral masks [2]. Roberg et al. present an envelope elimination and restoration (EER) approach, where a supply modulator is used to place an envelope on the transmitted waveform for spectral confinement, while maintaining saturation and the associated high-efficiency performance [3]. Rodriguez describes design and analysis of a power supply for envelope modulation [4]. Adaptive predistortion is presented as a solution for nonlinearity-related spectral spreading [5]. In these works, however, the ambiguity function is not discussed.

Spectrally confined waveforms are also considered. Faust demonstrates a method for generating spectrally confined waveforms. The autocorrelation of the waveforms are considered for the purpose of examining pulse compression properties [6]. This is a step toward consideration of the entire ambiguity function, because the time axis of the ambiguity function is the time-domain autocorrelation of the waveform. Autocorrelation is also considered by C.E. Cook in describing a method to reduce range sidelobe level [7], by M.R. Cook in examining continuous-phase modulation (CPM) [8], and by Jakabosky in optimizing polyphase-coded frequency modulation (PFCM) waveforms [9].

The effect of transmitters on waveforms and waveform optimizations has recently become an area of interest. Jakabosky has detailed a "transmitter-in-the-loop" optimization approach for continuous-phase modulation (CPM) [10] and orthogonal frequency division multiplexing (OFDM) [11] waveforms. It is suggested that radar transmitter distortion causes increased range sidelobes [10]. The results from [10] and [11] show that waveform optimization considering the transmitter nonlinear effects can provide decreased range sidelobe values in the time-domain autocorrelation results.

The present paper attempts to directly examine the impact of amplifier nonlinearities on the ambiguity function. A previous paper by our group examines ambiguity functions of measured amplifier output waveforms [12]. In the present paper simulation results are presented involving nonlinear amplifier models, and the artifacts of the nonlinearities, as manifested in the ambiguity function, are traced by examination of the theory. Section II discusses the expected impact of amplifier nonlinearities on the ambiguity function. Section III presents simulation results using nonlinear transistor models and resultant calculations of the ambiguity function. Section IV presents conclusions from these results. 


\section{SPeCtral SpREAdING AND the Ambiguity FunCtion}

A well-known result of amplifier nonlinearities is spreading of the spectrum [10]. This artifact is due to oddorder amplifier nonlinearities interacting on a multi-tone or broadband signal. The impact on the ambiguity function of these nonlinearities can be derived from the impact on the spectrum. If equation (1) is used to find the zero-delay part of the ambiguity function (the Doppler axis, where $\tau=0$ ), we obtain [13]

$$
\chi(0, u)=\int_{t=-\infty}^{\infty}|x(t)|^{2} e^{-j 2 \pi u t} d t=X(u) \star X(u)
$$

where the star, $\star$, denotes correlation

$$
X(u) \star Y(u)=\int_{u=-\infty}^{\infty} X(v) Y^{*}(v-u) d v
$$

The correlation of two functions is equal to the sum of the supports of the individual functions [14]. If, for example, $X(u)$ is zero for $|u|>A$ and $Y(u)$ is zero for $|u|>B$, then $X(u) \star Y(u)$ is zero for $|u|>A+B$.

Consider, then, the input spectrum to a nonlinear amplifier, $F(u)$, and the output spectrum from the amplifier, $Y(u)$. If the nonlinear amplifier causes spectral spreading, the support of $Y(u)$ will exceed that of $F(u)$. Thus, the support of $Y(u) \star Y(u)$ will exceed that of $F(u) \star F(u)$. Consequently, from $(2), \chi_{y}(0, u)$, will have a greater width along the $u$ axis than $\chi_{f}(0, u)$, the ambiguity function corresponding to $f(t)$.

The literature proposes that range sidelobes are increased as a result of nonlinearities [10]. Figure 1 shows the autocorrelation of a time-limited sinusoid and square wave (zero-Doppler cut of the ambiguity function). It can be seen that the range sidelobes are higher for the square wave. However, it must be noted that the square wave possesses greater energy than the sinusoid if both are of the same amplitude and frequency. Correspondingly, the main lobe is also higher for the square wave, as the ambiguity function value at zero range and Doppler is equal to the energy of the signal. If the autocorrelations are normalized by the signal energies, then the results are as shown in Fig. 2. In this case, the range sidelobes are actually slightly wider in the case of the sinusoid. The sidelobes of the square wave autocorrelation are triangular and fit inside the sinusoidal autocorrelation of the sinusoid. Both have the same amplitude. Because the center lobe of the ambiguity function has height equal to the energy of the associated waveform, it is expected that the main lobes would be of the same height when normalized to the signal energies (Fig. 2).

Figure 3 shows the magnitude spectra for the sinusoid and the square wave following normalization by the energy term. It can be seen that the square wave has lower main lobes and significant harmonics. The zero-range cut of the ambiguity function (along the Doppler axis) will be the autocorrelation of the spectrum, and as such, there will be significant additional lobes present along the Doppler axis for the square wave. While this considers the case of simple harmonic nonlinearities, amplifier third-order nonlinearities are expected to provide additional sidelobes close to the main lobe of the signal. For certain waveforms, third-order nonlinearities could cause the Doppler ambiguity to extend three times as far from the origin along the Doppler axis. This is a significant effect.



Fig. 1. Zero-Doppler cut (autocorrelation) of the ambiguity function for a time-limited sinusoid (solid line) and square wave (dashed line) of the same amplitude and frequency. The center lobe and sidelobes are larger for the ambiguity function of the square wave.

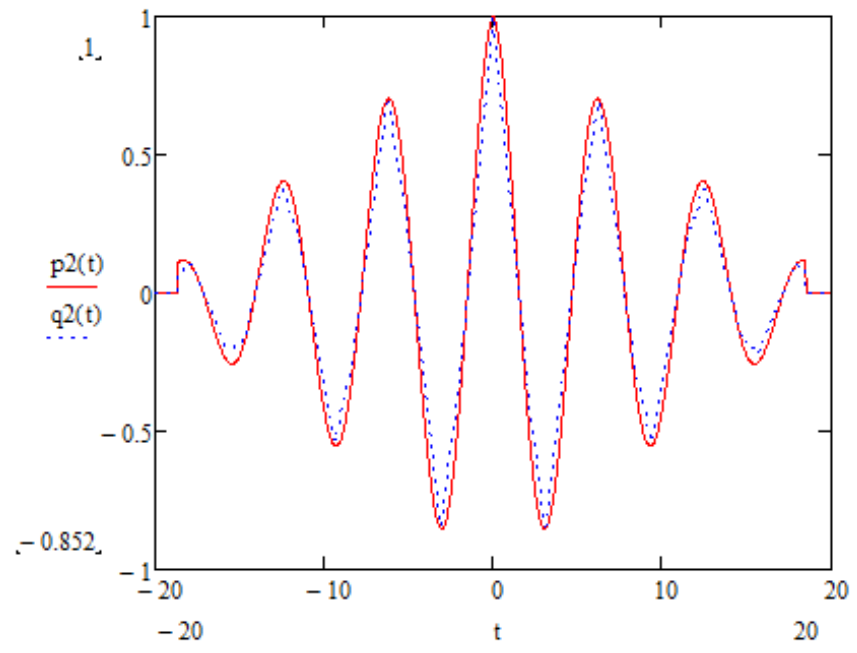

Fig. 2. Zero-Doppler cut (autocorrelation) of the ambiguity function for the time-limited sinusoid (solid line) and square wave (dashed line) after normalizing both ambiguity functions by the associated signal energy. 


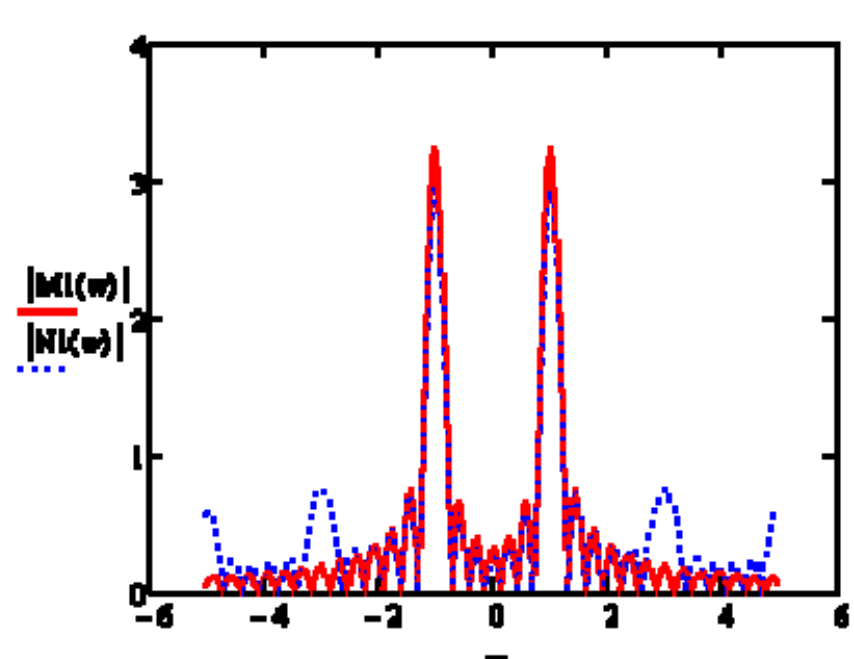

Fig. 3. Magnitude spectra for the sinusoid (solid lines) and square wave (dotted lines) of equal energy. Note that the square wave has distinguishable harmonic sidelobes.

\section{NONLINEAR AMPLIFIER CIRCUIT SIMULATIONS}

Circuit simulations were performed using the Advanced Design System (ADS) circuit simulator from Keysight Technologies. A built-in nonlinear field-effect transistor (FET) model was used for the simulations. The transistor was excited with a waveform that caused significant nonlinear effects in the output waveform, as might be encountered in operation in a power amplifier design. A seven-pulse Barker code with Gaussian pulses was used as the excitation waveform, as shown in Fig. 4. Figure 5 shows the waveform and spectrum of the output signal. As expected, the time-domain waveform is significantly flattened. The spectrum of Fig. 5(b) shows that the main lobe is narrower (this is because the time-domain waveform broadens), but that intermodulation distortion has created third- and fifth-order sidelobes that extend the range spectrum. The zero-Doppler slices (the values along the range axis) of the input and output waveform ambiguity functions are displayed in Figures 6 and 7, respectively. It can be seen that the results are very similar for the input and output waveforms. The zero-Doppler cut sidelobes of the output waveform appear flatter than the sidelobes for the input waveform, but the sidelobes seem to have almost exactly the same maximum height.

The zero-range slices of the input and output waveform ambiguity functions are displayed in Figures 8 and 9, respectively. Comparison of the decibel-scale results in Figures 8(b) and 9(b) reveals that ambiguity spikes of significant height appear at larger Doppler for the amplifier output waveform. Figure 10 shows a "heat-map" version of the ambiguity functions. Figure $10(\mathrm{a})$ is the ambiguity function for the amplifier input signal, and Figure 10(b) is the ambiguity function for the amplifier output signal. Comparing these figures shows that the height of the ambiguity is sustained further in the Doppler direction away from zero at all range values, and that the ambiguity sidelobes are wider in the range dimension for all values of Dopper.

To summarize, the following effects are caused by the amplifier nonlinearities: (1) spreading of the ambiguity in the Doppler dimension and (2) widening of the range sidelobes.

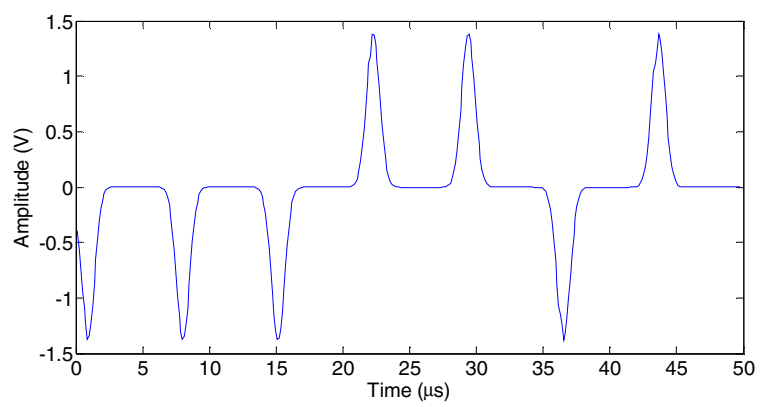

(a)

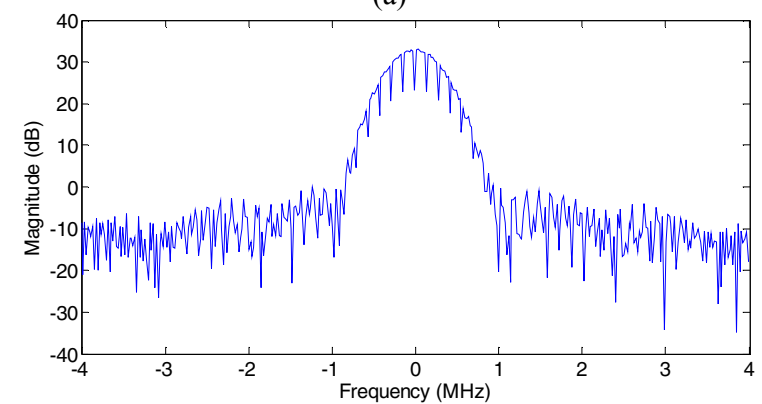

(b)

Fig. 4. Amplifier input (a) time-domain waveform and (b) spectrum for a seven-pulse Barker code.

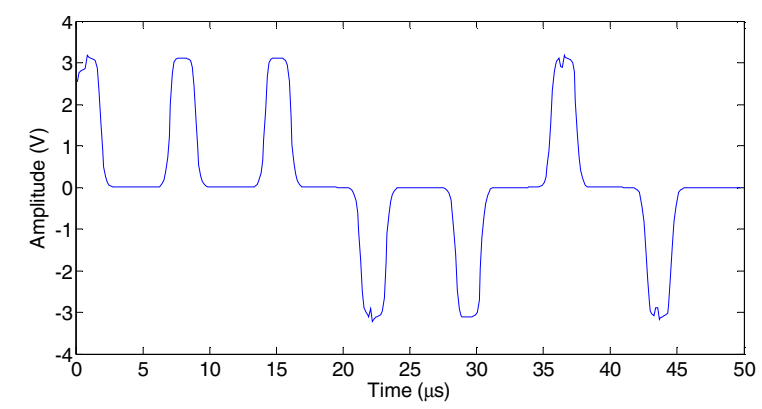

(a)

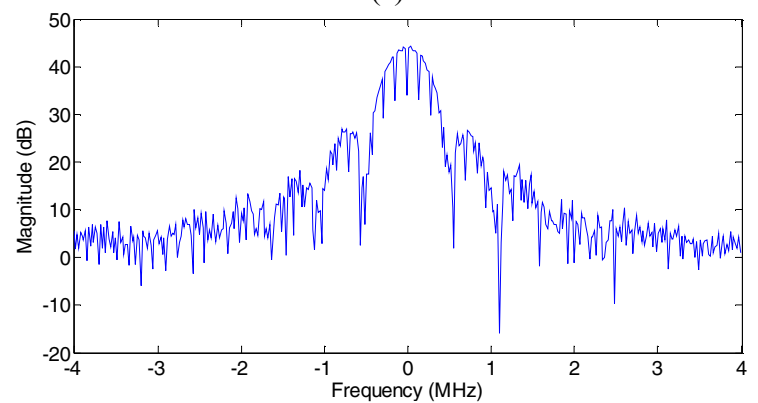

(b)

Fig. 5. Amplifier output (a) time-domain waveform and (b) spectrum for the seven-pulse Barker code. Effects of the nonlinearities can be observed in the flattening of the pulses in the time domain, the narrowing of the main spectral lobe in the frequency domain, and the additional spectral sidelobes in the frequency domain. 


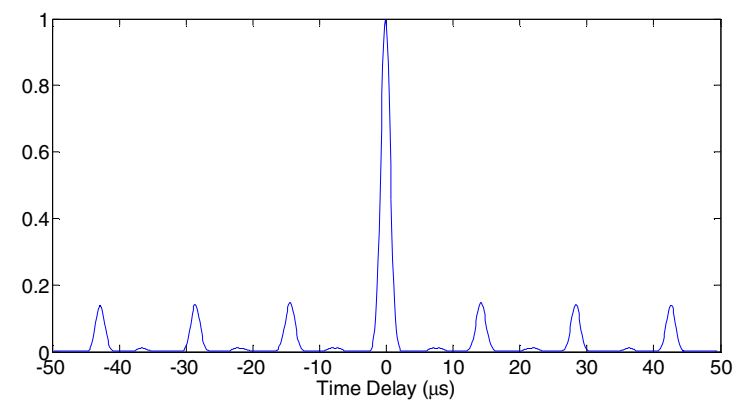

(a)

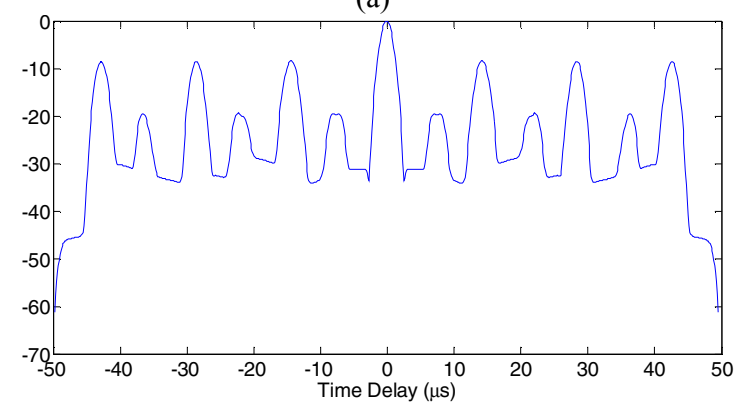

(b)

Fig. 6. Zero-Doppler slice of the ambiguity function for the amplifier input waveform in (a) linear scale and (b) decibel (dB) scale.

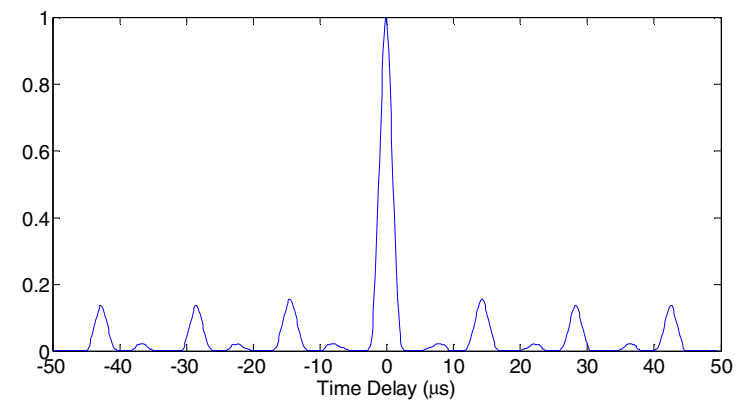

(a)

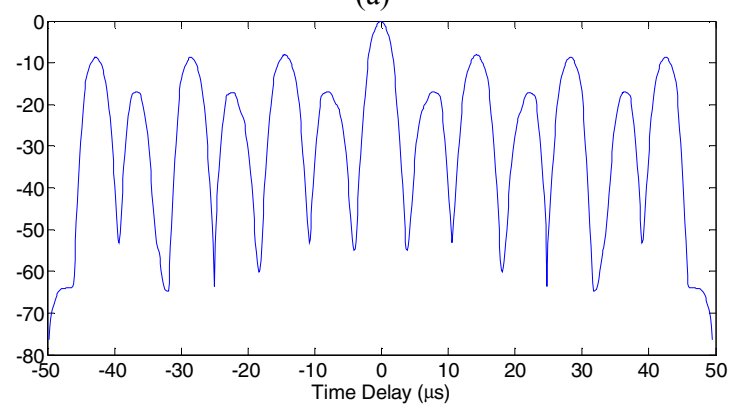

(b)

Fig. 7. Zero-Doppler slice of the ambiguity function for the amplifier input waveform in (a) linear scale and (b) decibel (dB) scale.

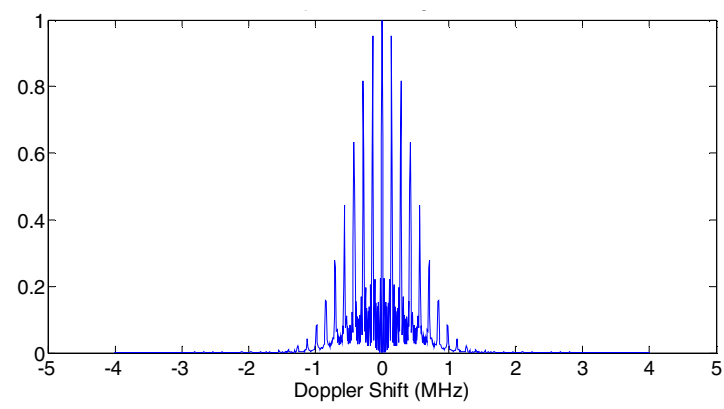

(a)

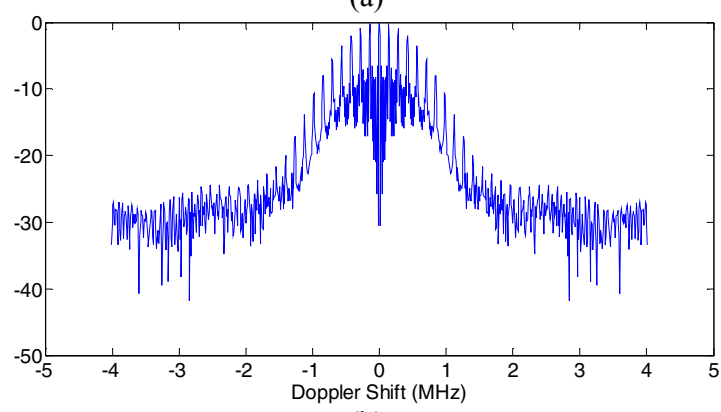

(b)

Fig. 8. Zero-range slice of the ambiguity function for the amplifier input waveform in (a) linear scale and (b) decibel (dB) scale.

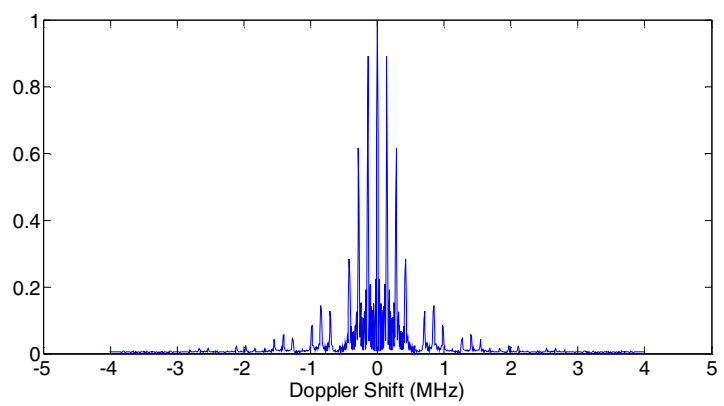

(a)

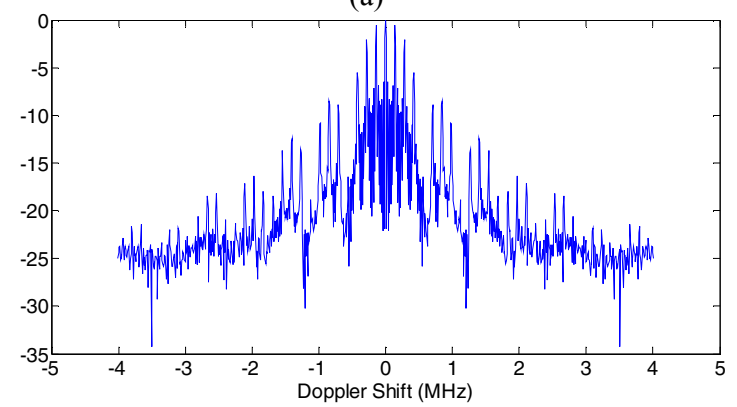

(b)

Fig. 9. Zero-range slice of the amplifier output waveform ambiguity function in (a) linear scale and (b) decibel (dB) scale. 


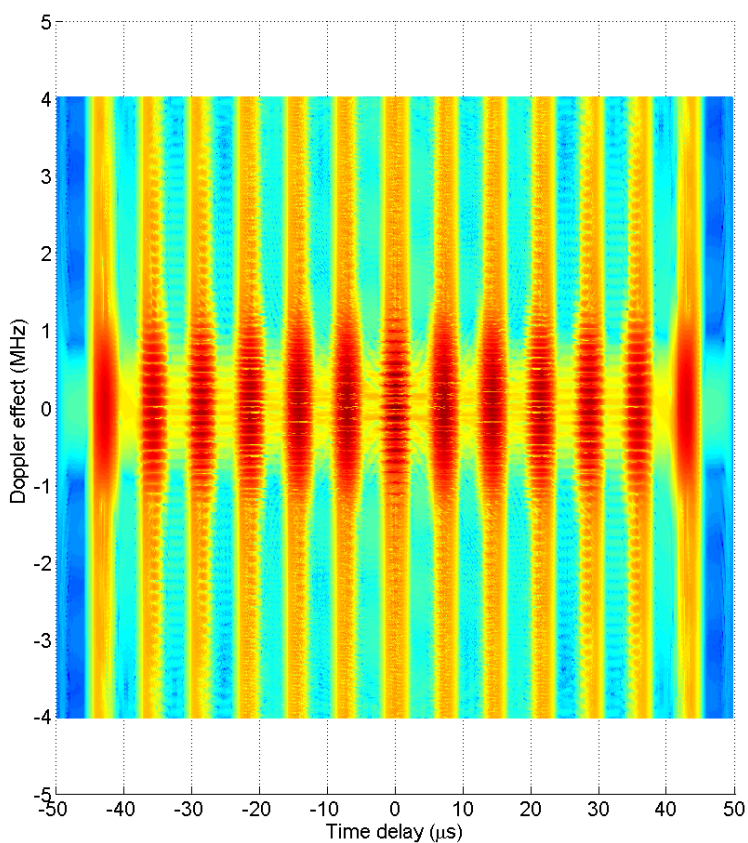

(a)

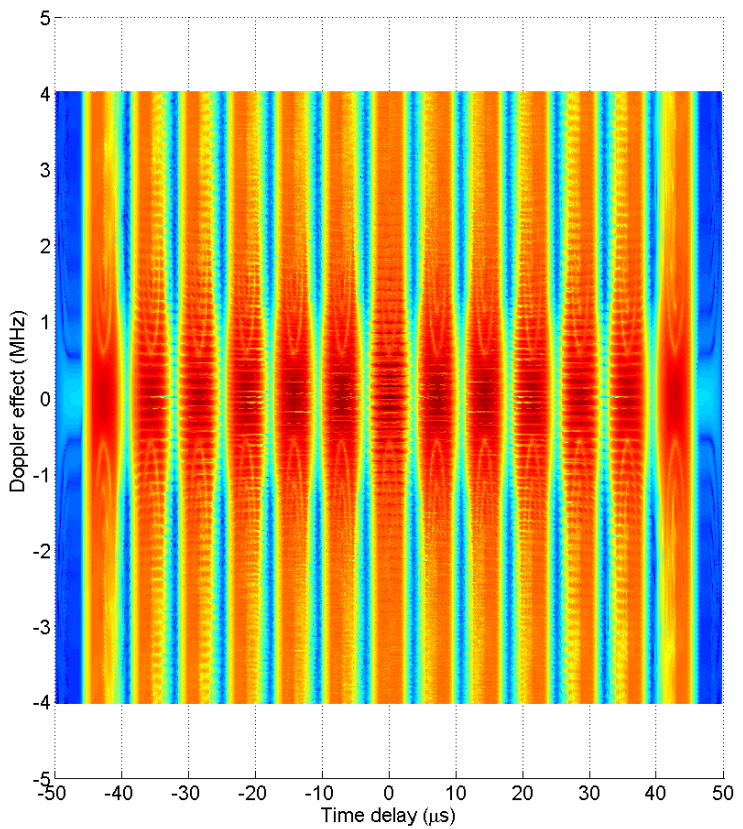

(b)

Fig. 10. Plots of the ambiguity function in "heat-map" format for (a) the amplifier input waveform and (b) the amplifier output waveform.

\section{CONCLUSIONS}

The ambiguity function is significantly affected by nonlinear power amplifiers used in radar transmitters. This is discussed through a theoretical and intuitive description, and is demonstrated by simulation of ambiguity functions for a simple baseband Gaussian-pulse Barker code. The effects on ambiguity functions from nonlinear amplifiers include (1) significant spreading of ambiguity in the Doppler dimension and (2) widening of the range sidelobes. The results from this paper will be useful in designing circuit and waveform optimizations for radar transmitters.

\section{ACKNOWLEDGMENTS}

This work has been funded under a grant from the National Science Foundation (Award Number ECCS-1343316). The authors would like to thank Keysight Technologies for costfree loan of the Advanced Design System simulation software.

\section{REFERENCES}

[1] M. Skolnik, Introduction to Radar Systems, Third Edition, McGrawHill, 2001.

[2] J. de Graaf, H. Faust, J. Alatishe, and S. Talapatra, "Generation of Spectrally Confined Transmitted Radar Waveforms: Experimental Results," 2006 IEEE Radar Conference, April 2006, pp. 76-83.

[3] M. Roberg, M. Rodriguez, D. Maksimovic, and Z. Popovic, "Efficient and Linear Amplification of Spectrally Confined Pulsed AM Radar Signals," IEEE Microwave and Wireless Components Letters, Vol. 22, No. 6, May 2012, pp. 279-281.

[4] M. Rodriguez, M. Roberg, A. Zai, E. Alarcon, Z. Popovic, and D. Maksimovic, "Resonant Pulse-Shaping Power Supply for Radar Transmitters," IEEE Transactions on Power Electronics, Vol. 29, No. 2, February 2014, pp. 707-718.

[5] A.E. Nordsjo, "An Algorithm for Adaptive Predistortion of Certain Time-Varying Nonlinear High-Power Amplifiers," 2002 International Radar Conference, Edinburg, United Kingdom, October 2002, pp. 469473.

[6] H.H. Faust, B. Connolly, T.M. Firestone, R.C. Chen, B.H. Cantrell, and E.H. Mokole, "A Spectrally Clean Transmitting System for Solid-State Phased Array Radars," 2004 IEEE Radar Conference, April 2004, pp. 140-144.

[7] C.E. Cook and J. Paolillo, "A Pulse Compression Predistortion Function for Efficient Sidelobe Reduction in a High-Power Radar," Proceedings of the IEEE, Vol. 52, No. 4, April 1964, pp. 377-389.

[8] S.D. Blunt, M. Cook, J. Jakabosky, J. de Graaf, and E. Perrins, "Polyphase-Coded FM (PCFM) Waveforms, Part I: Implementation," IEEE Transactions on Aerospace and Electronic Systems, Vol. 50, No. 3, pp. 2218-2229, July 2014.

[9] J. Jakabosky, S.D. Blunt, and B. Himed, "Optimization of 'OverCoded' Radar Waveforms," 2014 IEEE Radar Conference, Cincinnati, Ohio, May 2014, pp. 1460-1465.

[10] S.D. Blunt, J. Jakabosky, M. Cook, J. Stiles, S. Seguin, and E.L. Mokole, "Polyphase-Coded FM (PCFM) Waveforms, Part 2: Optimization," IEEE Transactions on Aerospace and Electronic Systems, Vol. 50, No. 3, pp. 2230-2241, July 2014.

[11] J. Jakabosky, L. Ryan, and S. Blunt, "Transmitter-in-the-Loop Optimization of Distorted OFDM Radar Emissions," 2013 IEEE Radar Conference, Ottawa, Ontario, Canada, April/May 2013.

[12] M. Fellows, C. Baylis, L. Cohen, and R.J. Marks II, "Calculation of the Radar Ambiguity Function from Time-Domain Measurement Data for Real-Time, Amplifier-in-the-Loop Waveform Optimization," Automatic RF Techniques Group (ARFTG) Conference, Columbus, Ohio, December 2013.

[13] R.J. Marks II, The Handbook of Fourier Analysis and Its Applications. Oxford University Press (2009)

[14] R.J. Marks II., John F. Walkup, and Marion O. Hagler. "A Sampling Theorem for Space-Variant systems." JOSA 66, no. 9 (1976): 918-921. 